\title{
The Efficacy and Safety of Modified Xiaoyao San for Perimenopausal Syndrome (PMS): A Systematic Review and Meta-Analysis
}

\author{
Wenzhi Hao1,2, Lian Gong1, Feifei Xue ${ }^{1 *}$ \\ ${ }^{1}$ School of Traditional Chinese Medicine, Jinan University, Guangzhou, China \\ ${ }^{2}$ Formula-Pattern Research Center, School of Traditional Chinese Medicine, Jinan University, Guangzhou, China \\ Email: haowenzhi@126.com, ^fayexue@126.com
}

How to cite this paper: Hao, W.Z., Gong, L. and Xue, F.F. (2019) The Efficacy and Safety of Modified Xiaoyao San for Perimenopausal Syndrome (PMS): A Systematic Review and Meta-Analysis. Journal of Biosciences and Medicines, 7, 60-72.

https://doi.org/10.4236/jbm.2019.74007

Received: March 15, 2019

Accepted: April 14, 2019

Published: April 17, 2019

Copyright ( 2019 by author(s) and Scientific Research Publishing Inc. This work is licensed under the Creative Commons Attribution International License (CC BY 4.0).

http://creativecommons.org/licenses/by/4.0/

\begin{abstract}
Objectives: To assess the efficacy and safety of modified Xiaoyao San (XYS) for treating Perimenopausal syndrome (PMS). Methods: Literature searches were carried out on PubMed, Cochrane Library, CNKI Database, Chinese Biomedical Literature Database, Wan Fang Database, and VIP Database up to December 2018. Hand search for further references was conducted. Study selection, data extraction, quality assessment, and data analyses were performed as request of the Cochrane standards. Results: Nine publications in total were suitable for inclusion. There was evidence that modified XYS was tested to be more effective in improving overall symptoms compared with HRT (odds ratio $3.50,95 \%$ CI 2.56 to 4.78 ). Whereas HRT was more sensitive and direct in decreasing FSH (WMD 6.69, 95\% CI 5.60 to 9.52) and LH (WMD 7.00, 95\% CI, 5.75 to 8.25 ) in comparison with XYS group. It was also strongly supported that XYS had less adverse effect than HRT (odds ratio $0.07,95 \% \mathrm{CI}$ 0.05 to 0.10 ). Conclusion: Modified XYS might be more effective and safer in treatment of perimenopausal syndrome. However, due to poor methodological quality in the majority of included studies, the potential benefit and safety about XYS need to be confirmed in rigorously designed, multi-centre, and large-scale trials.
\end{abstract}

\section{Keywords}

Xiaoyao San, Perimenopausal Syndrome (PMS), Meta-Analysis

\section{Introduction}

Perimenopausal syndrome (PMS) is a natural gynecological disease among women in reproductive age, and defined by the World Health Organization (WHO) as 
the permanent cessation of menstruation and a decrease in the levels of ovarian steroid hormones (estrogen and progesterone) due to the loss of ovarian follicular function. The final menstrual period is retrospectively assigned after 12 consecutive months of amenorrhea in the absence of other pathological or physiological causes [1] [2]. Women with PMS might suffer from the symptoms such as hot flashes and night sweats, insomnia, depression, vaginal dryness, mood disorders, fatigue or loss of energy and so on [3] [4], which have a quite negative impact on their daily lives. Around $75 \%$ of reproductive age women have mild symptoms and $20 \%-30 \%$ of women have clinically significant PMS symptoms [5] [6]. Additionally, 13\% - 18\% of women need treatment due to Premenstrual Dysphoric Disorder (PMDD), the severe form of PMS [7]. And prevalence tended to increase every year. [8]

PMS is most commonly treated with Estrogen replacement therapy (ERT), also called as Hormone replacement therapy (HRT) [9], which is a treatment of adjusting the menstrual cycle and alleviating the clinical symptoms by estrogen supplement [10]. However, the minimum effective dosage and potential side effects of HRT are still controversial [11]. HRT might be related to significantly higher breast cancer incidence [12], ovarian cancer risk [13], stroke and obstruction of a vein by blood clot risk [14], and also headache, weight gain, drowsiness, sexual dysfunction, and insomnia [15] [16]. As a result, compliance in women with PMS treated with HRT is rather poor. A telephone survey done in the US, reported $80 \%$ of women preferred non-pharmacological interventions, such as vitamins, cognitive behavioral therapy, complementary and alternative medicine to hormonal therapy such as estrogen therapy, combined oral contraceptives, pyridoxine, ethinylestradiol and drospirenone [17] [18] [19].

In traditional Chinese medicine (TCM), this syndrome is usually caused by decline of kidney qi, near exhaustion of reproductive substance (Tiangui) and disorder of the heart, liver and spleen as dysfunction of qi, blood, yin and yang. PMS is nearly equivalent to the TCM term "before and after menopause" which is divided into different syndromes according to various clinical symptoms and signs. Chinese herbal medicine has been a recent favorable therapeutic approach owing to the relative safety. Among all prescriptions, Xiaoyao San formula was most commonly used, accounting for $37.5 \%$ of all prescriptions [20].

Xiaoyao San (XYS) originated from the "prescription of the Bureau of Taiping People's Welfare Pharmacy" (Tai-Ping-Hui-Min-He-Ji-Ju-Fang) in the Song Dynasty, containing eight commonly used herbs (Bupleurum root, Chinese Angelica Root, White Peony Root, Poria, Atractylodes Rhizome, Roasted Ginger, Prepared Licorice Root, Menthol and Peppermint), has been used to treat mental disorders, menopathy and spleen gastric disease for centuries in China. Modified Xiaoyao formula usually refers to several formulas, whose basic ingredients are same as XYS, including Xiaoyao Wan (XYW), Danzhixiaoyao San (DZXYS), Jiaweixiaoyao San (JWXYS), Hexiaoyao San (HXYS), etc.

How about the efficacy of modified XYS in treatment of Perimenopausal syn- 
drome, and if it is really safer than HRT? With a view to answering these questions, the meta-analysis of randomized controlled trials evaluates the effects and safety of modified XYS in treating patients with PMS compared with HRT.

\section{Methods}

\subsection{Search Strategy}

Comprehensive literature searches were conducted in PubMed (1950-2018), Cochrane Library (1993 to December 2018), CNKI Database (1979 to December 2018), Chinese Biomedical Literature Database (1990 to December 2016), Wan fang Database (1982 to December 2018), and VIP Database (1989 to December 2018), and searched the reference list of retrieved papers. Keywords for searching included perimenopausal syudrome, before and after menopause, Climacteric syndrome and Xiaoyao Wan, Xiaoyao San, Xiaoyao powder. The search was restricted to studies carried out in humans. Papers published in English and Chinese were evaluated. There were no restrictions on population characteristics and publication type. Manual searches of conference compilations supplemented electronic searches. A recursive manual search of cited references in published studies was performed to identify other relevant studies.

\subsection{Study Selection}

Studies were considered to be eligible for inclusion if they met all of the following criteria: 1) Patients included in the study were diagnosed with Perimenopausal syndromes; 2) The study was performed as a randomized controlled trial (RCT); 3) XYS (all the modified Xiaoyao San formula were included) was compared with HRT; 4) Criteria for successful treatment were clearly clarified, and treatment success was not only measured in terms of the scores of Kupperman Index (KI) or Serum FSH, E2, HL, the criteria "recover, significant effective, effective, or not effective" was also included in the outcome measurement; 5) Treatment lasted for 3 weeks or more. For the meta-analysis, the terms of "patients without symptoms," Duplicated publications reporting the same groups of participants were excluded.

\subsection{Data Extraction}

Two researchers abstracted the data from the studies independently to avoid bias in the data abstraction process. All data were checked for internal consistency, and disagreements were resolved by discussion with other investigators. Details abstracted from the studies included the name of the first author, location of the report, year of publication, age of subjects in the XYS or HRT group, number of participants in each group, dosage of each intervention, duration of treatment, adverse effect and follow-up situation. Quality assessment of randomized controlled trials: sequence generation, allocation concealment, blinding of participants personnel and outcome assessors, incomplete outcome data, selective outcome reporting, and other sources of bias. 


\subsection{Data Analysis}

The statistical package Review Manager Software (RevMan 5.3) was used for data analyses, which was provided by The Cochrane Collaboration. Dichotomous data were presented as odds ratio (OR) and continuous outcomes as mean difference (MD), both with $95 \%$ confidence interval (CI). Meta-analysis was performed if the intervention, control, and outcome were the same or similar. The chi-squared test for heterogeneity was performed, and heterogeneity was presented as significant when I2 is over $50 \%$ or $\mathrm{P}<0.1$. Random effect model was used for the meta-analysis if there was significant heterogeneity; otherwise fixed effect model was used.

\section{Results}

\subsection{Search Results}

A total of 187 publications were identified by computer search and 4 by manual search of cited references. Of these, 4 articles were determined to be duplicated and other 137 studies were excluded because of not PMS or not PMS only, reviews and comments, Animal experiments, and so on. The remaining 50 reports were retrieved in full text, of which 41 were excluded for nonrandomized controlled reports or wrong RCT methods, untied with Chinese herb in control group or integrated with Estrogen in Experiment group, etc. Finally 9 studies [21]-[29] were satisfied the inclusion criteria for the meta-analysis (Figure 1), which were all published in Chinese.

Eight of the studies [21] [22] [23] [24] [25] [27] [28] [29] took comprehensive response effect as primary outcome on principle of National criterion of therapeutical effect [30], one used serum FSH, E2, LH as outcome [26]. Four of the studies treated PMS with Nilestriol in control group [22] [24] [28] [29].

Three used Medroxyprogesterone 17-acetate, respectively combined with Estradiol [21], Premarin [26], Stilbestrol [27]. Two trials had no details about HRT [23] [25]. A description of included trial characteristics can be found in Table 1.

\subsection{Characteristics and Methodological Quality of Included Trials}

The risk of bias assessment in the trials was summarized in Figure 2. A low risk of bias was found across studies for blinding of outcome assessment and incomplete outcome data, selective reporting. Two publications were of randomization procedures generated by random number tables [24] [25]. None of them have mentioned allocation concealment and double blind. One of them using serum hormone as outcomes, which would not be influenced by Measurement bias, was rated as low risk [24]. Four trials were considered as high risks ascribing to using decoction of herbal medicine in experiment and hardly achieving double blind [25] [26] [27] [28]. Two trials reported the follow-up but no dropouts presented [23] [26], five reported adverse reactions [21] [22] [26] [28] [29]. Among all trials, the characteristics of participants in different treatment groups were similar at baseline (age, sex, race, and disease course). 
W. Z. Hao et al.

Table 1. Characteristics of the 9 studies included in the meta-analysis.

\begin{tabular}{|c|c|c|c|c|c|c|c|c|}
\hline \multirow{2}{*}{ Author } & \multirow{2}{*}{$\begin{array}{l}\text { N (XYS } \\
\text { vs. HRT) }\end{array}$} & \multirow{2}{*}{$\begin{array}{l}\text { Age } \\
\text { (years) }\end{array}$} & \multirow{2}{*}{ Outcome } & \multicolumn{2}{|r|}{ Interventions } & \multirow{2}{*}{ - Duration } & \multirow{2}{*}{$\begin{array}{l}\text { Adverse } \\
\text { effects }\end{array}$} & \multirow{2}{*}{ Follow-up } \\
\hline & & & & Experimental & Control & & & \\
\hline Huang 2013 [21] & $48 / 44$ & 44.1 & $\begin{array}{l}\text { 1) Response rate } \\
\text { 2) single symptoms } \\
\text { improvement }\end{array}$ & XYW (TID) & $\begin{array}{c}\text { Estradiol (1 mg QD) + } \\
\text { Medroxyprogesterone (8 mg QD) }\end{array}$ & 8 weeks & $\mathrm{R}$ & N.R \\
\hline Tang 2005 [22] & $322 / 330$ & $45-55$ & Response rate & XYW (BID) & Nilestriol (1 - 2 mg BIW) & 8 weeks & $\mathrm{R}$ & N.R \\
\hline Zhang 2014 [23] & $21 / 21$ & 50 & Response rate & XYW (TID) & Estrogen (N.R.) & 3 weeks & N.R. & 3 months \\
\hline Hu 2009 [24] & $84 / 84$ & 49.6 & $\begin{array}{l}\text { 1) Response rate } \\
\text { 2) } \mathrm{FSH}, \mathrm{E} 2, \mathrm{LH}\end{array}$ & DZXYS (QD) & Nilestriol (2 mg BIW) & 12 weeks & N.R. & N.R \\
\hline $\begin{array}{l}\text { Yun et al. } 2015 \\
{[25]}\end{array}$ & $96 / 96$ & 49.7 & $\begin{array}{l}\text { 1) Response rate } \\
\text { 2) Kupperman Score }\end{array}$ & DZXYS (BID) & Estrogen and progesterone (N.R.) & 12 weeks & N.R. & N.R \\
\hline Liu 2008 [26] & $32 / 32$ & $40-60$ & $\begin{array}{l}\text { Single symptoms } \\
\text { improvement }\end{array}$ & DZXYS (QD) & $\begin{array}{c}\text { Premarin }(0.3 \mathrm{mg} \text { TID })+ \\
\text { Medroxyprogesterone }(10 \mathrm{mg} \text { QD })\end{array}$ & 4 weeks & $\mathrm{R}$ & N.R \\
\hline Zhou 1997 [27] & $120 / 60$ & N.R. & Response rate & DZXYS (QD) & $\begin{array}{c}\text { Stilbestrol (0.5 mg QD) + } \\
\text { Medroxyprogesterone (4 mg BID) }\end{array}$ & 12 weeks & N.R. & 2 years \\
\hline $\begin{array}{c}\text { Wang et al. } 2016 \\
{[28]}\end{array}$ & $40 / 40$ & $47-55$ & Response rate & JWXYS (TID) & Nilestriol (2 mg BIW) & N.R. & $\mathrm{R}$ & N.R \\
\hline Zhang 2011 [29] & $43 / 40$ & $42-54$ & Response rate & JWXYW (TID) & Nilestriol (2 mg BIW) & 8weeks & $\mathrm{R}$ & N.R \\
\hline
\end{tabular}

QD, once a day BID, twice a day TID, three times a day BIW, fortnightly, R, reported N.R., not report.

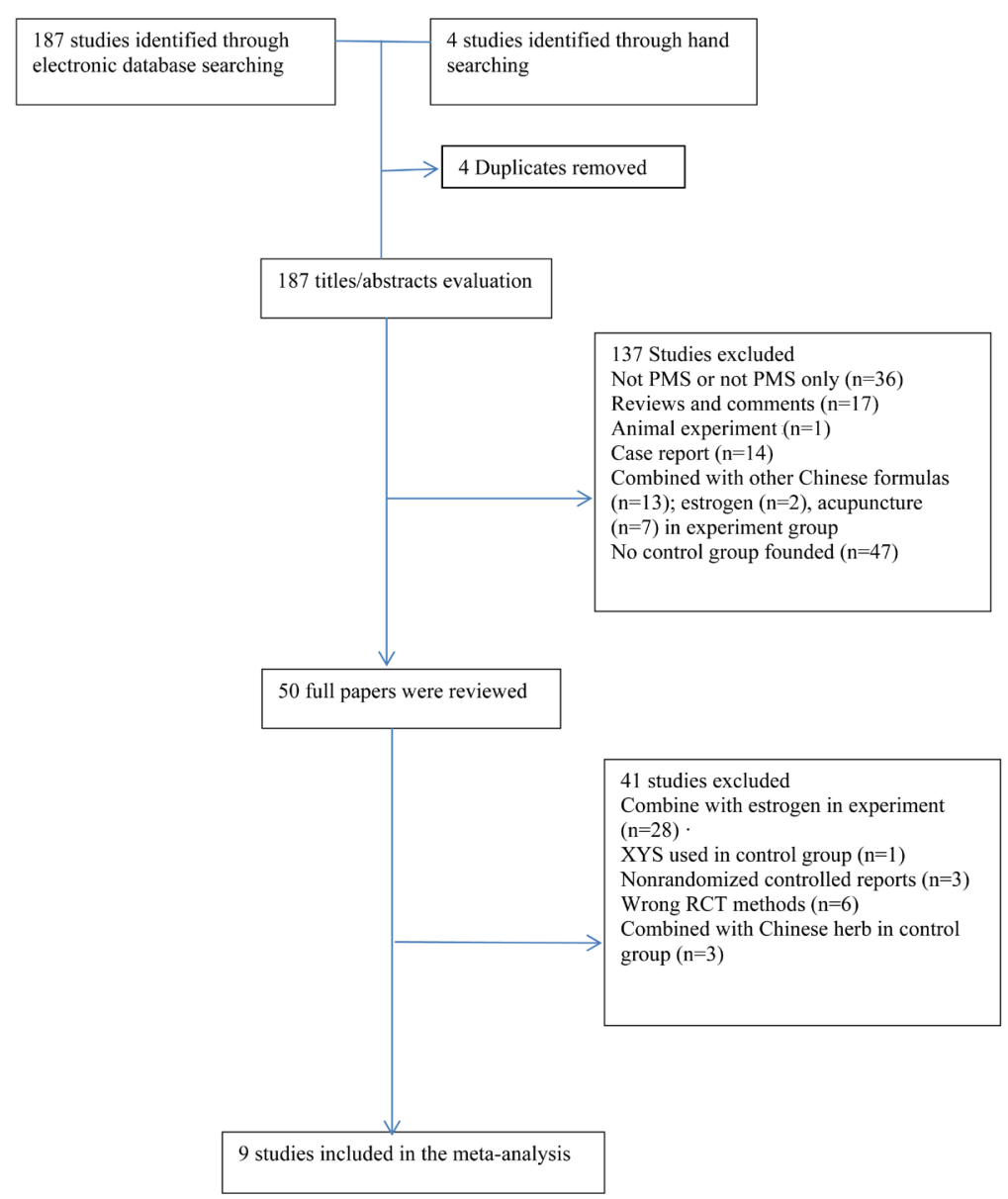

Figure 1. Flow chart of study selection process. 


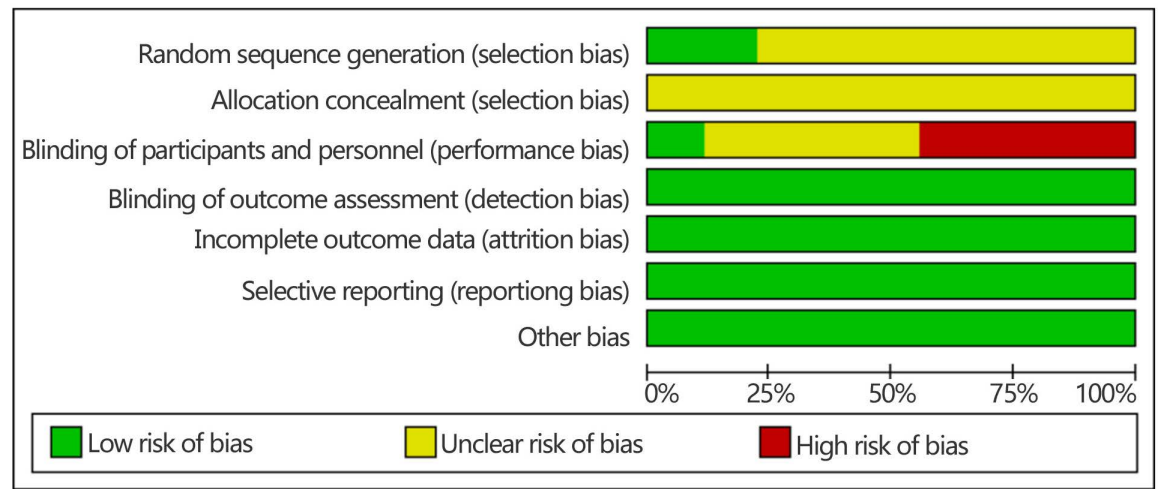

Figure 2. Risk of bias assessment.

\subsection{Analysis of XYS Formula}

Four kinds of XYS formula were used as intervention in experiment group. Three trials used fixed XYW throughout the treatment [21] [22] [23], one of them administered together with ginger water [22]. Four trials XYW was modified and combined with different Chinese herbs on the basis of syndrome differentiation [24]-[28], one used JWXYS [28] and one used DZXYS [29]. Interestingly we found JWXYS in the trial [28] was actually simply DZXYS. In Table 2, we listed the Chinese herbs in every prescription. Bupleurum root (Chai $\mathrm{Hu}$ ), Chinese Angelica Root (Dang Gui), White Peony Root (Bai Shao) showed up most often, and used in every prescription. The preparation formulation was divided into pills and decoction.

\subsection{Some Common Mistakes}

\subsubsection{Response Rate}

Eight randomized controlled trials evaluated response rate of modified XYS in comparison with HRT [21] [22] [23] [24] [25] [27] [28] [29]. Odds Ratio was used to assess the change of global symptoms in patients with PMS. Figure 3 showed the OR and summary OR for each trial. The summary OR was 3.50 (95\% CI: $2.56-4.78, \mathrm{P}<0.00001$ ), indicating that PMS patients with XYS treatment had more than 3 -fold higher probability of symptom relief compared to treatment with HRT. No substantial heterogeneity was found $(\mathrm{P}=0.29, \mathrm{I} 2=18 \%)$.

\subsubsection{Serum FSH, E2, LH}

Only one of the studies [24] performed the test of serum sexual hormone like FSH, E2, LH as outcomes. We found from the article that both the experiment group and control group could obviously decrease serum FSH and LH, while both had a tendency to increase serum E2, but no statistical significance existed. However, from the Meta-analysis it turned out that HRT showed a significant improvement in reducing FSH and LH by contrast with modified XYS. (FSH: WMD, 6.69; 95\% CI, 5.60 to 9.52; P < 0.00001, Figure 4(a); LH: WMD, 7.00; 95\% CI, 5.75 to $8.25 ; \mathrm{P}<0.00001$, Figure 4 (b)). E2 remained the same as what the article reported that no statistical significance was founded. (E2: WMD, 2.77; 95\% CI, -3.98 to $7.78 ; \mathrm{P}=0.42$, Figure $4(\mathrm{c})$ ) 
Table 2. The herbs used in the included 9 RCTs.

\begin{tabular}{|c|c|c|c|}
\hline \multirow[t]{2}{*}{ Trials included } & \multicolumn{3}{|c|}{ English herbal name(Chinese pinyin) in prescription in experiments } \\
\hline & & Regular herbs in XYS & Modified herbs in XYS \\
\hline \multicolumn{4}{|l|}{ Huang 2013} \\
\hline Tang 2005 & & \multicolumn{2}{|c|}{$\begin{array}{l}\text { White Atractylodes Rhizome (Bai Zhu) Poria (Fu Ling) Menthol and Peppermint (Bo He) } \\
\text { Roasted Ginger (Sheng Jiang) Prepared Licorice Root (Zhi Gan Cao) }\end{array}$} \\
\hline \multicolumn{4}{|l|}{ Zhang 2014} \\
\hline Liu 2008 & & $\begin{array}{l}\text { White Atractylodes Rhizome (Bai Zhu) } \\
\text { Poria (Fu Ling) Prepared Licorice Root (Zhi } \\
\text { Gan Cao) }\end{array}$ & $\begin{array}{l}\text { Tree Peony Root Bark (Mu Dan Pi) Cape Jasmine Fruit (Zhi Zi) } \\
\text { Prepared Rehmannia Root (Shu Di Huang) Asiatic Cornelian } \\
\text { Cherry Fruit (Shan Zhu Yu) Glossy Privet Fruit (Nv Zhen Zi) } \\
\text { Barbary Wolfberry Fruit (Gou Qi Zi) }\end{array}$ \\
\hline Hu 2009 & $\begin{array}{l}\text { Bupleurum root } \\
\text { (Chai } \mathrm{Hu})\end{array}$ & $\begin{array}{l}\text { White Atractylodes Rhizome (Bai Zhu) } \\
\text { Poria (Fu Ling) Prepared Licorice Root (Zhi } \\
\text { Gan Cao) }\end{array}$ & $\begin{array}{l}\text { Tree Peony Root Bark (Mu Dan Pi) Cape Jasmine Fruit (Zhi Zi) } \\
\text { Prepared Rehmannia Root (Shu Di Huang)Asiatic Cornelian } \\
\text { Cherry Fruit (Shan Zhu Yu)Glossy Privet Fruit (Nv Zhen Zi) }\end{array}$ \\
\hline & Angelica Root & & Yerba-Detajo Herb (Han Lian Cao) \\
\hline Yun et al. 2015 & White Peony & $\begin{array}{l}\text { Poria (Fu Ling) } \\
\text { Prepared Licorice Root (Zhi Gan Cao) }\end{array}$ & $\begin{array}{l}\text { Tree Peony Root Bark (Mu Dan Pi) Cape Jasmine Fruit (Zhi Zi) } \\
\text { Bone Fossil of Big Mammals (Long Gu) Oyster Shell (Mu Li) } \\
\text { Silktree Albizia Bark (He Huan Pi) Germinated Barley (Xiao Mai) }\end{array}$ \\
\hline Zhou 1997 & Root (F & $\begin{array}{l}\text { White Atractylodes Rhizome (Bai Zhu) } \\
\text { Poria (Fu Ling) Menthol and Peppermint (Bo } \\
\text { He) Prepared Licorice Root (Zhi Gan Cao) } \\
\text { Roasted Ginger (Sheng Jiang) }\end{array}$ & Tree Peony Root Bark (Mu Dan Pi) Cape Jasmine Fruit (Zhi Zi) \\
\hline Zhang 2011 & & $\begin{array}{l}\text { White Atractylodes Rhizome (Bai Zhu) } \\
\text { Poria (Fu Ling) Menthol and Peppermint (Bo } \\
\text { He) Prepared Licorice Root (Zhi Gan Cao) }\end{array}$ & Tree Peony Root Bark (Mu Dan Pi) Cape Jasmine Fruit (Zhi Zi) \\
\hline $\begin{array}{l}\text { Wang et al. } \\
\quad 2016\end{array}$ & & $\begin{array}{l}\text { White Atractylodes Rhizome (Bai Zhu) } \\
\text { Menthol and Peppermint (Bo He) }\end{array}$ & $\begin{array}{l}\text { Morinda Root (Yin Yang Huo) Chinese Magnoliavine Fruit (Wu } \\
\text { Wei Zi) Prepared Rehmannia Root (Shu Di Huang) }\end{array}$ \\
\hline
\end{tabular}

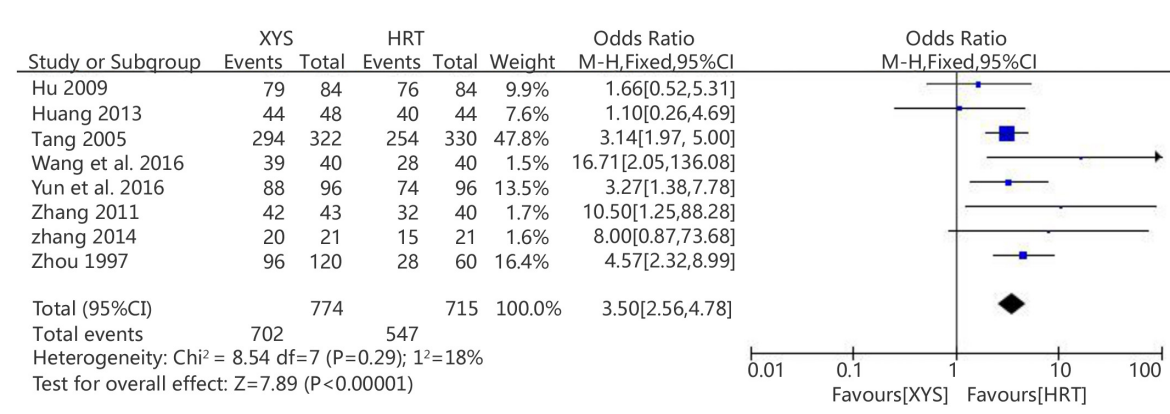

Figure 3. Forest plot of response rate. XYS vs. HRT.

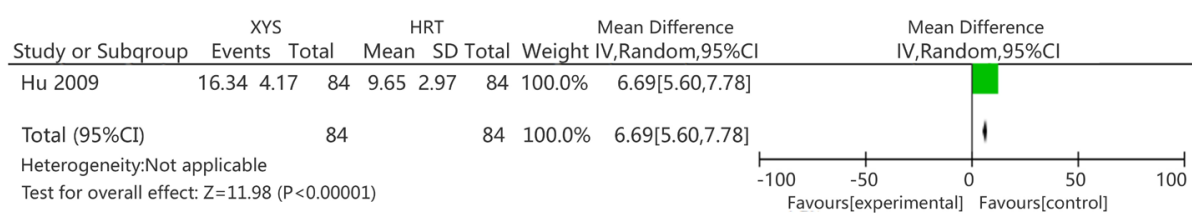

(a)

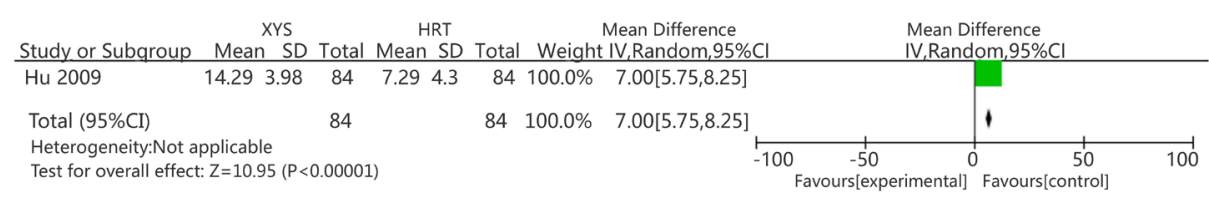

(b) 


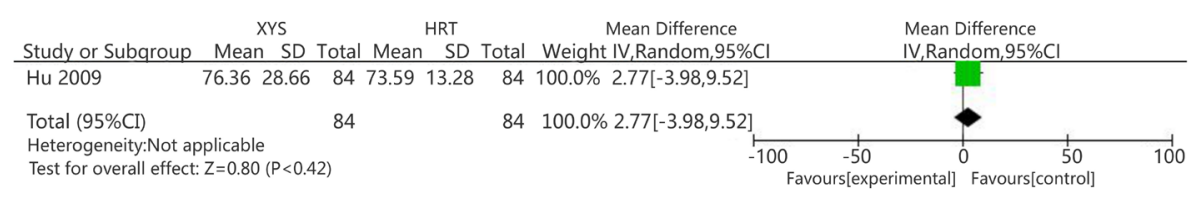

(c)

Figure 4. (a) Serum FSH; (b) Serum LH; (c) Serum E2.

\subsubsection{Adverse Events}

Six trials out of nine included studies mentioned the occurrence of adverse events [21] [22] [24] [26] [28] [29]. One of them reported no adverse effects during treatment of both groups [24]. Three trials declared no serious adverse events found in modified XYS group; whereas the patients experienced HRT had suffered from gastroenteric adverse reaction including nausea and vomiting [21] [28] [29]. Two trials reported a variety of symptoms developed such as nausea, headache, edema, leukorrhagia, and abdominal distention, colporrhagia in both modified XYS groups and HRT groups [22] [26]. Meta-analysis robustly supported that XYS showed less side effects than HRT, and had statistical significance. (OR 0.07; 95\%, CI $0.05-0.10 ; \mathrm{P}<0.00001$, Figure 5)

\section{Discussion}

The results of the present meta-analysis revealed that modified Xiaoyao San was superior to hormone replacement therapy in improving comprehensive PMS symptoms (OR 3.50; point; 95\% CI: 2.56 - 4.78). The study also demonstrated that adverse events after treated with XYS were significantly lower than with HRT supported by Meta-analysis (OR 0.07; 95\%, CI 0.05 - 0.10).

In TCM, the basic mechanism of Perimenopausal syndrome is deficiency of the kidney. However, effective treatment cannot be guaranteed only by nourishing kidney in treating PMS. The reason would be that PMS is intimately related to the dysfunction of Liver. Liver depression and Qi stagnation leading to transformation of Fire and disturbing cardiac sprit, or invasion of Liver Qi into the Spleen and Stomach are also crucial mechanism of PMS [30] [31] [32].

In accordance with the mechanism of PMS, herbs of modified Xiaoyao San in the trials included can be divided into several groups. The first group is composed of Adix Bupleuri (Chai Hu), Chinese Angelica and White Peony Root (Bai Shao), which plays the main role in whole formula. Adix Bupleuri (Chai $\mathrm{Hu}$ ) contributes to Soothing liver and relieving depression, the experiment stated that the methanolic Bupleurum falcatum (BFM) extract had dose-dependent possibility of antidepressant-like activity valuable to alternative therapy for depression and that the mechanism of action might involve the serotonergic and noradrenergic systems [33]. Chinese Angelica (Dang Gui) can be used for tonifying and harmonizing blood, regulating menstruation to relieve pain. Biochemically, Angelica can promote hematopoietic function of bone marrow and spleen cells and significantly increase the number of hemoglobin and red blood cells [34], can also potentiate the immune functions [35]. The function of White Peony 


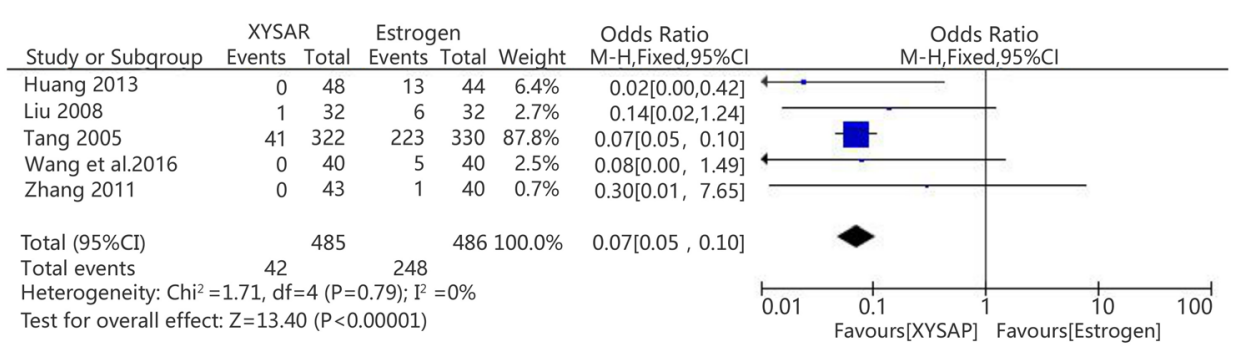

Figure 5. Forest plot of adverse events. XYS vs. HRT.

Root (Bai Shao) focuses on liver, it can remarkably disperse liver. Studies suggested treatment of the rats with White Peony Root can significantly suppressed the depression-like behavior and increased brain BDNF levels in CORT-treated rats [36]. And the modulation of the hypothalamic-pituitary-adrenal axis and up-regulation of serotonergic and noradrenergic systems might be important mechanisms underlying the antidepressant-like effects of paeoniflorin in CUS-treated rats [37]. A combination of these three herbs used most frequently in prescriptions was demonstrated to significantly soothes liver, nourish blood and regulate nutrient [38]. The second group, White Atractylodes Rhizome (Bai Zhu), Poria (Fu Ling), Prepared Licorice Root (Zhi Gan Cao) are conducive to strengthen spleen, and then Tree Peony Root Bark (Mu Dan Pi), Cape Jasmine Fruit (Zhi $\mathrm{Zi}$ ) are used for relieving depression and clearing heat, Prepared Rehmannia Root (Shu Di Huang), Asiatic Cornelian Cherry Fruit (Shan Zhu Yu), Glossy Privet Fruit (Nv Zhen Zi), Yerba-Detajo and Herb (Han Lian Cao) replenish kidney-yin, Morinda Root (Yin Yang Huo) is for warmly invigorating kidney yang, Bone Fossil of Big Mammals (Long Gu), Oyster Shell (Mu Li), Silktree Albizia Bark (He Huan Pi), Germinated Barley (Xiao Mai) calm the nerves. A combination of various single herbs aims to restore the dysfunction of Liver depression and Qi stagnation, readjust the balance of the whole body [39] and produce a holistic effect through multi-level, multi-target and multi-channel control.

It can be affirmed that the safety of XYS appears satisfactory. It is noteworthy XYS has been approved as a non-prescription formula by State Food and Drug Administration (SFDA), which reinforce validity about the safety of XYS. The explanation of the encouraging safety might be that the multi-target effect of herbal preparations may produce relatively low concentration of active ingredients [40]. As we mentioned above that majority of women with PMS preferred non-pharmacological interventions to HRT [17] [18] [19], XYS can be recommended for its distinguished safety.

From the meta-analysis, hormone replacement therapy was more sensitive and direct than modified XYS in ameliorating the sexual hormone such as FSH and LH, but no statistically significant difference between two groups in increasing serum E2. Nevertheless, there was only one study involved, the result needs further clinical trials or laboratory bench studies to verify.

We should consider several limitations before accepting the findings of this 
paper. First, the quality of the included studies was generally not high. No multi-center, large sample, and cooperative studies were found, and most of the trials were of small sample size, and no studies estimated the sample size. Most of the studies did not report about allocation concealment process, which might have created potential selection bias. And it's been difficult to implement double blind for distinct intervention dosage forms between two groups. Therefore, measurement bias was always of concern. Second, given the fact that all of the nine trials included were in Chinese, the publication bias possibly existed. Third, different modified Xiaoyao prescriptions and various forms and dosage were used in the trials, medication frequency were from once a day to three times a day, and the treatment duration varied from 4 weeks to 12 weeks.

\section{Conclusion}

In conclusion, the results of the present paper preliminarily confirm that modified Xiaoyao San is more efficacious and has less adverse events compared to hormone replacement therapy in treatment of perimenopausal syndrome. However, considering the general quality of the reports was moderate; the effectiveness and safety of Xiaoyao San prescription need further rigorous trials, multi-centre, large-scale, and transnational cooperative RCTs to prove.

\section{Acknowledgements}

This research was supported by the National Natural Science Foundation of China (No. 81503496 and 81603520), the Natural Sciences Foundation of Guangdong province (No. 2016A030310084), the Administration of Traditional Medicine of Guangdong province (No. 20141211, 20150430 and 20161063), the Fundamental Research Funds for the Central Universities (No. 21616315).

\section{Conflicts of Interest}

The authors declare no conflicts of interest regarding the publication of this paper.

\section{References}

[1] Jaspers, L., van Dijk, G.M., Roeters van Lennep, J.E., Franco, O.H., Kavousi, M., et al. (2015) Health in Middle-Aged and Elderly Women: A Conceptual Framework for Healthy Menopause. Maturitas, 81, 93-98. https://doi.org/10.1016/j.maturitas.2015.02.010

[2] Hulka, B.S. and Meirik, O. (1996) Research on the Menopause. Maturitas, 23, 109-112. https://doi.org/10.1016/0378-5122(95)00967-1

[3] Hartlage, S.A., Freels, S., Gotman, N., et al. (2012) Criteria for Premenstrual Dysphoric Disorder: Secondary Analyses of Relevant Data Sets. Archives of General Psychiatry, 69, 300-305. https://doi.org/10.1001/archgenpsychiatry.2011.1368

[4] El Shafie, K., Al Farsi, Y., Al Zadjali, N., et al. (2011) Menopausal Symptoms among Healthy, Middle-Aged Omani Women as Assessed with the Menopause Rating Scale. Menopause, 18, 1113-1119. https://doi.org/10.1097/gme.0b013e31821b82ee 
[5] Campbell, E.M., Peterkin, D., O’Grady, K. and Sanson-Fisher, R. (1997) Premenstrual Symptoms in General Practice Patients. Prevalence and Treatment. The Journal of Reproductive Medicine, 42, 637-646.

[6] Borenstein, J., Chiou, C.F., Dean, B., et al. (2005) Estimating Direct and Indirect Costs of Premenstrual Syndrome. Journal of Occupational and Environmental Medicine, 47, 26-33. https://doi.org/10.1097/01.jom.0000150209.44312.d1

[7] Halbreich, U., Borenstein, J., Pearlstein, T., et al. (2003) The Prevalence, Impairment, Impact, and Burden of Premenstrual Dysphoric Disorder (PMS/PMDD) Psychoneuroendocrinology, 28, 1-23. https://doi.org/10.1016/S0306-4530(03)00098-2

[8] Goldbacher, E.M., Bromberger, J. and Matthews, K.A. (2009) Lifetime History of Major Depression Predicts the Development of the Metabolic Syndrome in Middle-Aged Women. Psychosomatic Medicine, 71, 266-272. https://doi.org/10.1097/PSY.0b013e318197a4d5

[9] Genazzani, A.R. and Genazzani, A.D. (2015) Polycystic Ovary Syndrome: From Contraception to Hormone Replacement Therapy. In: Fauser, B.C.J.M. and Genazzani, A.R., Eds., Frontiers in Gynecological Endocrinology, ISGE Series, Springer, Cham, Switzerland, 25-31.

[10] Panay, N., Hamoda, H. and Arya, R. (2013) The 2013 British Menopause Society \& Women's Health Concern Recommendations on Hormone Replacement Therapy. Menopause International, 19, 59-68. https://doi.org/10.1177/1754045313489645

[11] Li, C.C., Wang, J.J., Chen, C., et al. (2013) Treating Menopause Syndrome by Kuntai Capsuale and Hormone Replacement Therapy: A Meta-Analysis of Efficacy and Safety Comparison. Chinese Journal of Integrated Traditional Chinese and Western Medicine, 33, 1183-1190.

[12] Lundqvist, A., Andersson, E., Ahlberg, I., et al. (2016) Socio-Economic Inequalities in Breast Cancer Incidence and Mortality in Europe: A Systematic Review and Meta-Analysis. European Journal of Public Health, 26, 804-813. https://doi.org/10.1093/eurpub/ckw070

[13] Li, K., Hüsing, A., Fortner, R.T., et al. (2015) An Epidemiologic Risk Prediction Model for Ovarian Cancer in Europe: The EPIC Study. British Journal of Cancer, 112, 1257-1265. https://doi.org/10.1038/bjc.2015.22

[14] Boardman, H.M., Hartley, L., Eisinga, A., et al. (2015) Hormone Therapy for Preventing Cardiovascular Disease in Both Healthy Post-Menopausal Women and Post-Menopausal Women with Pre-Existing Cardiovascular Disease. Cochrane Database of Systematic Reviews, No. 3, CD002229.

[15] Marjoribanks, J., Brown, J., O’Brien, P.M., et al. (2013) Selective Serotonin Reuptake Inhibitors for Premenstrual Syndrome. Cochrane Database of Systematic Reviews, No. 3, CD001396.

[16] Hu, X.H., Bull, S.A., Hunkeler, E.M., et al. (2004) Incidence and Duration of Side Effects and Those Rated as Bothersome with Selective Serotonin Reuptake Inhibitor Treatment for Depression: Patient Report versus Physician Estimate. Journal of Clinical Psychiatry, 65, 959-965. https://doi.org/10.4088/JCP.v65n0712

[17] O’Brien, P.M. (1993) Helping Women with Premenstrual Syndrome. BMJ, 307, 1471-1475. https://doi.org/10.1136/bmj.307.6917.1471

[18] DeMonico, S.O., Brown, C.S. and Ling, F.W. (1994) Premenstrual Syndrome. Current Opinion in Obstetrics and Gynecology, 6, 499-502.

https://doi.org/10.1097/00001703-199412000-00005

[19] Kim, S.Y., Park, H.J. and Lee, H. (2011) Acupuncture for Premenstrual Syndrome: 
A Systematic Review and Meta-Analysis of Randomized Controlled Trials. BJOG: An International Journal of Obstetrics \& Gynaecology, 118, 899-915. https://doi.org/10.1111/j.1471-0528.2011.02994.x

[20] Hsing, Y., Chen, B.-S., Huang, Y., et al. (2014) Identifying Chinese Herbal Medicine for Premenstrual Syndrome: Implications from a Nationwide Database. BMC Complementary and Alternative Medicine, 14, 206. https://doi.org/10.1186/1472-6882-14-206

[21] Huang, Z.-J. (2013) The Clinical Observation of the Treatment of Premenstrual Syndrome with Xiaoyao Wan. Practical Journal of traditional Chinese Medicine, 29, 86-86.

[22] Tang, B.-P. and Long, C.-X. (2005) Treatment of 322 Cases of Perimenopausal Syndrome with Xiaoyao Pill. Hunan Journal of Traditional Chinese Medicine, 21, 54.

[23] Zhang, W.-G. (2014) Clinical Observation on Treatment of Climacteric Syndrome with Xiaoyao Pill. China Practical Medicine, 9, 193.

[24] Hu, K.-J. (2009) The Treatment on 84 Cases Perimenopausal Syndrome with Danzhi Xiaoyao San. Modern Distance Education of Chinese Medicine, 7, 27-28.

[25] Yun, Q. and Fu, Y. (2015) The Treatment on Perimenopausal Syndrome with Danzhi Xiaoyao San. Jilin Journal of Traditional Chinese Medicine, 35, 495-497.

[26] Liu, X.-Y. (2008) Clinical Observation on Treatment of 64 Cases Perimenopausal Syndrome with Danzhi Xiaoyao San. Guiding Journal of Traditional Chinese Medicine, 14, 47-48.

[27] Zhou, R.-Q. (1997) Clinical Research on Treatment of Spleen Deficient and Liver Depression Type Menopausal Syndrome with Danzhi Xiaoyao Powder in the Treatment of Accumulate Heat. Guangming Journal of traditional Chinese Medicine, 4, 48-50.

[28] Wang, X.-Y. and Yu, J.-W. (2016) To Explore the Clinical Effect of Modified Xiaoyao Pill in the Treatment of Climacteric Syndrome. Guide of China Medicine, 14, 13-14.

[29] Zhang, H. (2011) Application of Modified Xiaoyao Pill in the Treatment of Climacteric Syndrome. Guiding Journal of Traditional Chinese Medicine, 08, 92.

[30] State Administration of Traditional Chinese Medicine (1994) Diagnostic Criteria of TCM Symptoms. Nanjing University Press, Nanjing, 6.

[31] Li, J. (2004) Concealment Methods of Clinical Random Allocation. Chinese Evidence-Based Medicine, 4, 714-715

[32] Li, R.-X., Ma, M. and Xiao, X.-R. (2016) Perimenopausal Syndrome and Mood Disorders in Perimenopause: Prevalence, Severity, Relationships, and Risk Factors. Medicine (Baltimore), 95, e4466. https://doi.org/10.1097/MD.0000000000004466

[33] Kwon, S., Lee, B., Kim, M., et al. (2010) Antidepressant-Like Effect of the Methanolic Extract from Bupleurum Falcatum in the Tail Suspension Test. Progress in Neuro-Psychopharmacology and Biological Psychiatry, 34, 265-270. https://doi.org/10.1016/j.pnpbp.2009.11.015

[34] Zhang, X.-J., Zhu, C.-C. and Hu, L. (2002) The Immune Activity of Angelica Sinensis Polysaccharide and Its Effect on Hematopoietic Function. Pharmacology and Clinics of Chinese Materia Medica, 18, 24-25.

[35] Han, S.B., Kim, Y.H., Lee, C.W., et al. (1998) Characteristic Immunostimulation by Angelan Isolated from Angelica Gigas Nakai. Immunopharmacology, 40, 39-48. https://doi.org/10.1016/S0162-3109(98)00026-5 
[36] Mao, Q.-Q., Huang, Z., Ip, S.-P., Xian, Y.-F. and Che, C.-T. (2012) Peony Glycosides Reverse the Effects of Corticosterone on Behavior and Brain BDNF Expression in Rats. Behavioural Brain Research, 227, 305-309.

https://doi.org/10.1016/j.bbr.2011.11.016

[37] Qiu, F.-M., Zhong, X.-M., Mao, Q.-Q. and Huang, Z. (2013) Antidepressant-Like Effects of Paeoniflorin on the Behavioural, Biochemical, and Neurochemical Patterns of Rats Exposed to Chronic Unpredictable Stress. Neuroscience Letters, 541, 209-213. https://doi.org/10.1016/j.neulet.2013.02.029

[38] Zhang, C.-Y., Tang, Y.-X., Zeng, N., et al. (2009) Xiaoyao Powder and Its Formula for the Effect of Behavioral Depression Model Rats. Journal of Chengdu University of Traditional Chinese Medicine, 32, 51-53.

[39] Chou, P.B., Morse, C.A. and Xu, H. (2008) A Controlled Trial of Chinese Herbal Medicine for Premenstrual Syndrome. Journal of Psychosomatic Obstetrics \& Gynecology, 29,185-192. https://doi.org/10.1080/01674820801893011

[40] Shen, X., Zhao, Z.-Y., Luo, X., Wang, H., Hu, B.-X. and Guo, Z.-H. (2016) Systems Pharmacology Based Study of the Molecular Mechanism of SiNiSan Formula for Application in Nervous and Mental Diseases. Evidence-Based Complementary and Alternative Medicine, 2016, Article ID: 9146378.

https://doi.org/10.1155/2016/9146378 